\title{
Lead advancement in pediatric patients with select secure 3830 pacing leads
}

\begin{abstract}
Select Secure 3830 pacemaker leads have been shown to be safer and more advantageous in the short term for pediatric pacemaker patients compared to previous pacemaker lead designs. The two case studies outlined below illustrate potential pitfalls in the medium to long term care of patients with this pacing lead. Further research on the long term risks/benefits of these leads must be performed to determine the long term functionality of the Select Secure 3830 lead.
\end{abstract}

Keywords: pediatrics, pacing leads, select secure 3830, lead advancement
Volume I Issue 3 - 2014

\section{MichaelW Rudokas, Meghna D Patel,Tammy M Bowman, George FVan Hare, Jennifer N \\ A Silva}

Washington University School of Medicine/St. Louis Children's Hospital, USA

\section{Correspondence: Jennifer NA Silva, I Children's Place, 8th floor NWT - CB 81 16, Saint Louis, MO 63110, USA, Tel 314- 454-6095,Email rudokas_m@kids.wustl.edu}

Received: July 03, 2014 | Published: July 07, 2014

\section{Introduction}

The SelectSecure3830 steroid eluting, bipolar, non-retractable screw-in transvenous lead (Medtronic, Inc.) provides a thinner diameter lead for pacemaker patients due to the lack of a central stylet lumen. This $4.1 \mathrm{~F}$ lead has been shown in preliminary data to be both safe and efficacious ${ }^{1}$ without increasing the risk of venous thrombosis. ${ }^{2}$ Long term safety results for the lead are still pending. Pediatric pacemaker patients present a unique challenge to lead placement as they have significant linear growth often requiring leads to be advanced or exchanged. Lead extraction/advancement has the chance for major complications particularly with older leads. ${ }^{3}$

\section{Case report}

We report our institutional experience with lead advancement with the Select Secure 3830 lead in two pediatric patients. In the first case, a 5-year-old female with a history of complete heart block underwent implantation of a single chamber transvenous pacemaker with a Medtronic 3830-59 SelectSecure ventricular pacing lead at the age of 3. She was brought back to the EP lab for advancement of her 2 yearold ventricular lead due to growth related ventricular lead tension. Under fluoroscopic guidance, the lead was easily advanced into the vasculature gaining several inches. During that same procedure, the patient was upgraded to a dual chamber system with a second Medtronic $3830-59 \mathrm{~cm}$ lead placed in the atrium.

The second case was a 12-year-old female with history of complete heart block who underwent placement of a dual chamber transvenous pacemaker at 8 years of age with two Medtronic $3830-59 \mathrm{~cm}$ pacing leads. She returned to the EP laboratory with 4yearold leads due to growth related tension of the atrial lead. Under fluoroscopic guidance, the atrial lead was advanced further into the right atrial appendage with some difficulty. The following morning, her atrial lead was found to be non-functional but intact. Attempts to either advance or extract the lead with simple traction were unsuccessful, and as such a new $3830-59 \mathrm{~cm}$ lead was implanted in the right atrial appendage leaving sufficient slack for growth. The ventricular lead was unable to be advanced due to scar tissue at the innominate vein/SVC junction. Follow-up at 1 month showed stable atrial pacing/sensing thresholds.

\section{Discussion}

Previous research on the Select Secure 3830 lead has been focused on safety during implantation and short/medium term follow up. The results of our case studies show that through the medium and long term this lead will be subject to the usual problems of transvenous leads and may not be easily advanced as hoped. The lead inconsistencies started to appear with longer term follow up, at 4 years in one of our cases. A definitive conclusion on the risks and benefits of lead advancement/ extraction with the SelectSecure 3830 lead cannot be concluded from the reported cases. More studies are needed to evaluate the long term functionality of the SelectSecure 3830 stylet-less lead.

\section{Acknowledgement}

None.

\section{Conflicts of interest}

Authors declare that there are no conflicts of interest.

\section{References}

1. Gammage MD, Lieberman RA, Yee R, et al. Multi-center clinical experience with a lumenless, catheter-delivered, bipolar, permanent pacemaker lead: implant safety and electrical performance. Pacing Clin Electrophysiol. 2006;29(8):858-865.

2. Gabbarini F, Agnoletti G. Selective-site pacing in paediatric patients: use of the Select Secure System and risk of vein occlusion. Europace. 2019;12(9):1286-1289.

3. Cecchin F, Atallah J, Walsh EP, et al. Lead extraction in pediatric and congenital heart disease patients. Circ Arrhythm Electrophysiol. 2010;3(5):437-444. 